\title{
THE ROLE OF THE CIMSTEEL INTEGRATION STANDARDS IN AUTOMATING THE ERECTION AND SURVEYING OF STRUCTURAL STEELWORK
}

\author{
Kent A. Reed \\ Leader, Computer Integrated Construction Group \\ Building Environment Division \\ Building and Fire Research Laboratory \\ National Institute of Standards and Technology \\ Gaithersburg, MD 20899-8630 \\ http://cic.nist.gov \\ kent.reed@nist.gov
}

\begin{abstract}
The second release of the CIMsteel Integration Standards (CIS/2) has been endorsed by the American Institute of Steel Construction as the standard for the electronic exchange of structural steel project information for the North American steel design and construction industry. Derived from the deliverables and experiences of the Pan-European Eureka CIMsteel Project and published by the Steel Construction Institute in the UK, the CIS/2 was developed with a life-cycle view of structural steelwork information in mind. However, the primary focus of both developers and implementers of $\mathrm{CIS} / 2$ has been on the pre-construction processes of design, analysis, detailing, and shop fabrication. This paper examines the applicability of CIS/2 to on-site construction processes, focusing on automating the erection and surveying of structural steelwork and integrating these two processes into the overall project delivery system.
\end{abstract}

KEYWORDS: automated construction, integration, product data model, project delivery, structural steelwork

\section{Introduction}

Successful integration of an automated process into a project delivery system depends on the ability to describe the desired result of the process in a manner understandable to its automation technology. Similarly, it depends on the ability of the automation technology to describe the actual result of the process in a manner understandable to subsequent processes. In this paper, the second release of the CIMsteel Integration Standards (CIS/2) [1] is examined for its ability to specify these descriptions in the specific cases of automating the erection of new structures and the surveying of existing structural steelwork.

The CIS/2 has been endorsed by the American Institute of Steel Construction (AISC) as the standard for the electronic exchange of structural steel project information for the North American structural steel design and construction industry [2]. The CIS/2 is derived from the deliverables and experiences of the Pan-European Eureka CIMsteel project and is intended to specify the exchange of data and the integration of engineering applications across steelwork design, analysis, and manufacturing.

A number of software vendors with structural steelwork applications are developing and demonstrating CIS/2-compliant translators for their products as part of the AISC Electronic Data Interchange initiative [3]. Initial case studies have shown significant benefits resulting from the use of these translators to exchange data between software applications in real projects. These benefits include knowing earlier in the project timeline approximately how much and what types of steel need to be reserved, reducing the time needed to estimate the material cost as part of the bidding process, and 
saving weeks of detailing time in a typical project [4].

The CIS/2 characterization of steelwork is intended to cover the needs of on-site construction as an aspect of manufacturing. However, the emphasis in the CIS/2 documentation is clearly on the pre-construction processes of design, analysis, detailing, and shop fabrication. None of the existing implementation efforts specifically addresses the information needs of field erection, although some do involve applications that can assist construction planning (for example, through the breakdown of the structure into erection zones and the identification by zone of the members to be shipped).

In order to automate the field erection of a structure as an integrated process in the overall project delivery system, one needs to access computer-sensible descriptions (1) of the parts and prefabricated assemblies that are to be delivered to the construction site, (2) of the sequence in which these parts and assemblies are to be assembled during erection to create the final structure, (3) of the joint systems connecting these pieces together, and (4) of the positions and orientations of all the pieces in their final locations. Automated construction equipment can then proceed to erect the structure without human direction.

Likewise, to automate the surveying of an existing structure as an integrated process, one needs a computer-sensible description of a model of the steel pieces composing the structure that can be populated with piece identification and position data acquired in the survey using various metrology systems.

In the following sections, the ability of the CIS/2 to provide these descriptions and thus to support the automation and integration of the erection and surveying of structural steelwork is explored.

\section{Overview of CIS/2}

The following is an extremely brief overview. The specification [1] should be consulted for details about what is said here, and for all the topics that aren't discussed here, such as the definitions of features and fastener mechanisms.

The CIS/2 was developed using some of the methodologies and technologies of the international product data standard ISO 10303 [5], known familiarly as STEP. The information requirements for relevant concepts in the domain of structural engineering were captured in structured English. After these information requirements were agreed, they were interpreted in a formal, computer-sensible model known as the Logical Product Model (which is LPM/5 in the second release of CIS). The information constructs in LPM/5 are defined as a schema using the ISO/STEP information modeling language known as EXPRESS [6]. This schema represents the constructs in terms of their underlying data entity types, attributes, relationships among entity types, and constraints.

An implementation of CIS $/ 2$ in a software application proceeds first by developing mappings between the concepts represented in the application and like concepts defined in the LPM/5. Depending on the particular concept(s) involved, a mapping may be as simple as one-toone or as complicated as many-to-many with associated constraints. Translators for importing and exporting information to and from the application in the form of CIS/ 2 exchange files are developed based on these mappings and the ISO/STEP clear text encoding method [7]. This method establishes the syntax of the exchange file and the rules for composing exchange structures in that syntax. It should be noted that the exchange structures can not be interpreted without recourse to the LPM/5 which establishes their meanings.

The LPM/5 supports three principal views of structural steelwork information, represented as analysis models, design models, and manufacturing models. Data representing one, two, or all three views may be present in an exchange file.

Analysis models of the structure are built up from nodes and elements and support a number 
of different static and dynamic analysis methods. Further, analysis results may be given at nodes, element ends, or at a point within an element, and the results can be described in a variety of ways.

Design models represent the structure as a design assembly for the purpose of member and connection design. Design assemblies can be decomposed into other design assemblies and ultimately into design parts and design joint systems, which are the conceptual representations of a basic piece of steel and a basic joint system, respectively. Design results for members and connections can be described in terms of elastic or plastic resistance.

Manufacturing models represent the structure as manufacturing assemblies for the purpose of detailing, production planning, and manufacturing. Located assemblies are built up from located parts and located joint systems, which are the representations of a basic physical piece of steel and of a basic physical joint system (e.g., a fastener group or a weld), respectively. In turn, all these located items can be built up into larger located assemblies, culminating in the complete structure. Features also are located with respect to the item they modify.

The located parts and located joint systems and their higher order located assemblies represent the items that actually occur in the completed structure. The material and geometrical definitions of these items are separated into part, joint system, and manufacturing assembly definitions that are referenced by their respective located items. This separation of data facilitates the economical reuse of generally verbose definition data across many occurrences of what are often identical items.

Although the LPM/5 supports the explicit description of geometry using the ISO/STEP geometric resources [8], most CIS/2 parts are expressed using implicit descriptions of geometry based on the specification of a transverse section profile (or a sheet width) and a longitudinal length through which the section profile is swept.
The LPM/5 defines a hierarchical system of locations (e.g., positions and orientations) such that an item is located with respect to the higher order item to which it belongs. For example, a feature may be located with respect to a part, which in turn may be located with respect to an assembly, and so on up to the location of a structure with respect to a site and the location of the site with respect to the earth. Each location is defined using a specialization of the coord_system entity. For most CIS/2 manufacturing model instantiations, this specialization turns out to be the coord_system_cartesian_3d entity, which encapsulates the ISO/STEP geometrical entity axis2_placement_3d [8]. This entity has among its attributes a 3D point defining the origin of the coordinate system and a set of vectors defining the $3 \mathrm{D}$ orientation of the coordinate system.

The preceding information constructs allow the expression of information about structural steelwork in the form of data instances. They don't allow the expression of information about that data, e.g., of metadata related to its management over the life of a project. The Data Management Control (DMC) schema in the LPM/5 provides that capability. The DMC concepts are important to this study because they allow one to exchange and manage multiple versions of data instances. For example, they could be used to maintain simultaneously an "as designed" model of a structure, an "as built" model, and a current "as is" model, with the data instances interrelated in a manner similar to the content of a software change control and configuration management system. The details of the DMC are given in the CIS/2 [1]. For the purpose of this study, it is sufficient to know that the DMC schema allows the representation of such information as the unique identifier for a data item, the person who created the data item, whether the data item is new or has been modified, why it was modified, and the date of modification.

\section{CIS/2 Applicability to Automated Erection}

It should already be apparent from the preceding overview that the $\mathrm{CIS} / 2$ is applicable to the 
automated erection of new structures. Through the composition hierarchy of the manufacturing model, all the parts and prefabricated assemblies that are to be delivered to the construction site can be described, along with the joint systems connecting these pieces together. Through the nested coordinate systems that locate parts in assemblies and locate parts and assemblies into bigger assemblies, and so on up to locating a structure on a site, the positions and orientations of the pieces and all their features can be defined with respect to a geo-referenced global coordinate system. Of course, the global coordinate system used in the manufacturing model and the global coordinate system used in the automated erection process must be reconciled before proceeding with erection.

One complication that arises in using the LPM/5 manufacturing model is its extensive use of implicit geometry. A member may be represented by a manufacturing assembly composed of several plates welded to a wideflange section with a number of clip angles on the section and on the plates. As stated in the overview, each of these parts---plate, section, and clip angle---is normally represented implicitly through the specification of a transverse section profile and a longitudinal length. Any datum of interest, sometimes called a principal point or a fiducial point, on the member must be calculated from these specifications and the (possibly) nested coordinate systems. However, this is really just a detail to be taken care of in software.

Another complication that arises is the need to be able to map between a piece physically present on the site and the representation of the same piece in the manufacturing model. A unique piece mark can serve to identify the two items but this is insufficient to ensure the items are oriented the same way in both worlds. An obvious way to ensure this orientation is to create a rule-based convention for placing the piece mark in a specific location on the physical item. This is similar to the rule-based conventions found in software that lays out members in a common way in $2 \mathrm{D}$ fabrication drawings, such that the left end and the front face of the member as shown in the drawing always bear the same relationship to the member.

Finally, the LPM/5 location mechanism is based on the STEP axis2_placement_3d entity, which encapsulates the $3 \mathrm{D}$ point of origin and the $3 \mathrm{D}$ orientation of a Cartesian coordinate system. With some automated placement techniques, this is just the right information. With others, the information needed may be, e.g., the set of 3D points defining all the corners of the member. Again, this is a detail to be taken care of in software.

\section{CIS/2 Applicability to Automated Surveying}

Two different situations arise in the surveying of existing structural steelwork. In the first, a CIS/2 representation of the structure in the form of a manufacturing model already exists. It might be the "as required" model provided by the project design team, for example. The member identification and location data measured in the survey can be used in this situation to update the existing CIS/2 representation to document the "as is" condition of the structure. Automation of this process would be relatively straightforward.

If the surveying application supports the DMC schema, then this "as is" representation could be achieved economically using DMC constructs to record the survey measurements with respect to their equivalent measures in the existing model. This process can be thought of as the electronic version of "red lining" a drawing. With DMC implemented, even fragmentary survey measurements can be associated properly with the model.

If the DMC schema is not supported in the survey application, then the existing CIS/2 representation would have to be duplicated and the copy updated to account for the survey measurements. Because it results in an entirely new model, this is a less desirable approach. It is a particularly undesirable approach if less than 100 percent of the structure is surveyed. In this case it becomes difficult to build a consistent model because the CIS/2 manufacturing model does not provide any versioning capability and 
hence can not make a distinction between original and updated location values.

Of course, the global (presumably georeferenced) coordinate systems used in the existing manufacturing model and in the survey process must be reconciled before any model updating can occur. As well, the nested coordinate systems present in the manufacturing model must be taken into account properly. This nesting is dependent on the composition hierarchy used in creating the manufacturing model and that hierarchy may not be apparent to the survey process. Hence, the most transparent approach would appear to be to make the survey measurements in the global coordinate system and transform the location data for each survey datum in the manufacturing model to the same global coordinate system for comparison. As described in the previous section, the datum may have to be computed from an implicit geometrical description of the item in question. For each datum, the inverse of the transform then can be used to transform the relevant survey measurement back into the nested coordinate systems as required for updating the model. Finally, all survey data of parts and assemblies must be reduced to the LPM/5 location mechanism, based on the axis2_placement_3d entity, which encapsulates a 3D position and a $3 \mathrm{D}$ orientation as opposed to a collection of $3 \mathrm{D}$ positions of the end points of a member.

In the second situation arising in surveying, a CIS/2 representation of the structure does not already exist but must be created from the survey measurements. This task could be accomplished directly in the CIS $/ 2$ representation with a suitable new software application. Equally, the application could be an existing structural steelwork detailing package with a CIS/2 translator. Either way, the surveying application must build up a model of the parts and assemblies in the structure from detailed measurements of the members without a priori knowledge of the nature of the members, making automation of the surveying process difficult. In this situation, however, the composition hierarchy is known explicitly and the location data can be transformed appropriately.

\section{General Issues}

Establishing that the information structures needed to support construction site processes are present in the LPM/5 is a necessary but not sufficient condition for success. These information structures must be implemented in the CIS/2 translators for the attendant software applications. The CIS/2 use conformance classes to document implementation requirements. The conformance classes already developed in the $\mathrm{CIS} / 2$ need to be reviewed for adequate coverage of construction-related information requirements and then used to specify the required translator capabilities.

In common with most significant product data standards, the LPM/5 sometimes provides more than one way to specify the same information. Where found to be present, these redundancies must be circumscribed through implementers' agreements so that misinterpretation of exchanged information is minimized.

Also in common with most significant product data standards, the LPM/5 contains many weakly specified uses of character strings as identifiers, labels, and descriptions. Again, where these are found to be present in the information structures needed to support construction site processes, they should be strongly specified in implementers' agreements so that misinterpretation of exchanged information is minimized. As an example of such an agreement, albeit at the national level rather than the level of individual implementers, the AISC has updated its naming convention for structural steel products for use in electronic data interchange so that software implementers no longer have to decide for themselves how to construct character strings to designate standard sections.

Finally, the use of CIS/2 to integrate automated processes in the construction of structural steelwork will still fail despite attention to all of the above general issues if the users of the CIS/2-enabled software applications don't 
provide the necessary information. As an example, the CIS/2 allow for the use of georeferenced coordinate systems, and the conformance class approach taken in CIS/2 can be used to ensure the translators involved properly map to and from the LPM/5 representation of this information, but none of this can force the user correctly to geo-reference the manufacturing model of a structure, and virtually no one does today using their favorite structural steelwork detailing system. A series of recommended practices will need to be developed to guide the end user to ensure success.

\section{Summary}

The second release of the CIMsteel Integration Standards has been endorsed by the American Institute of Steel Construction as the standard for the electronic exchange of steel project information for the North American structural steel design and construction industry. In this paper, the applicability of the CIS/2 to on-site construction processes has been assessed, focusing in particular on the erection of new structures and the surveying of existing structural steelwork. The manufacturing model view of CIS $/ 2$ has been found to be capable of supporting these processes. With the ongoing implementation effort in the AISC Electronic Data Interchange initiative and with the development of modest new applications, these processes can be automated and integrated into the overall project delivery system using CIS/2 as the baseline specification.

\section{REFERENCES}

1. Crowley, A.J., and Watson, A. S., CIMsteel Integration Standards Release 2, Vol.1-6, The Steel Construction Institute, Ascot UK, 2000. (see also the companion website http://www.cis2.org)

2. Press release, American Institute of Steel Construction, Chicago, IL, 2001 (reprinted in Foreword of $\mathrm{CIS} / 2$ ). (see also the website http://www.aisc.org)
3. Hamburg, S. E., and Holland, M. V., Leaping Ahead with EDI, Modern Steel Construction, Vol. 39, No. 1, pp. 42-48, American Institute of Steel Construction, Chicago, IL, February 1999. (see also http://www.aisc.org/edi.html)

4. Electronic Data Interchange Case Studies, The American Institute of Steel Construction, Chicago, IL, 2001. (electronic version available from http://www.aisc.org/edi.html)

5. ISO 10303-1, Industrial automation systems Product data representation and exchange - Part 1: Overview and fundamental principles, ISO/IEC Geneva, Switzerland, 1994.

6. ISO 10303-11, Industrial automation systems - Product data representation and exchange Part 11: Description methods: The EXPRESS language reference manual, ISO/IEC Geneva, Switzerland, 1994. (with Technical Corrigendum 1, 1999)

7. ISO 10303-21, Industrial automation systems - Product data representation and exchange Part 21: Implementation methods: Clear text encoding of the exchange structure, ISO/IEC Geneva, Switzerland, 2002.

8. ISO 10303-42, Industrial automation systems - Product data representation and exchange Part 21: Integrated generic resource: Geometric and topological representation, ISO/IEC Geneva, Switzerland, 2000. (with Technical Corrigendum 1, 2001). 